\title{
Infección Urinaria en Niños y Niñas internados: Características Clínicas y Microbiológicas
}

\section{Urinary Infection in Children and boarding Girls: clinical features and Microbiological}

Dra. María José Sánchez", Dra. Dolores Lovera², Prof. Dr. Antonio Arbo ${ }^{2},{ }^{1}$ Hospital Central de las Fuerzas Armadas ${ }^{2}$ Instituto de Medicina Tropical

La infección del tracto urinario (ITU) es una de las principales causas de infección bacteriana en niños. Un diagnóstico oportuno, un tratamiento adecuado $y$ un seguimiento estrecho previenen el daño renal crónico. En este trabajo se revisan las características clínicas y microbiológicas de los niños y niñas menores de 15 años ingresados en el Hospital Central de las Fuerzas Armadas (HCFFAA) desde enero de 2011 a noviembre de 2013 mediante la revisión de las historias clínicas. Se encontró un total de 35 casos con aislamiento positivo de urocultivo, donde la Prevalencia se mantiene casi similar, con promedio de 12 casos por año (3\% del ingreso hospitalario), la edad más afectada fue la de los lactantes menores de 2 años, el sexo femenino predomina en todas las edades con una relación de 4:1 con respecto a los varones. Los síntomas más frecuentes fueron la fiebre $y$ el vómito.

Al igual que en otros estudios la Escherichia coli es el germen de mayor aislamiento $y$ el tratamiento de elección son las cefalosporinas de tercera generación. Tres pacientes han presentado complicaciones, como consecuencia hicieron urosepsis.

Palabras claves: Cefalosporinas, daño renal crónico, urosepsis.

Abstract

Background: Urinary tract infection (UTI) is a major cause of bacterial infection in children. An early diagnosis, appropriate treatment and close monitoring prevent chronic kidney damage. In this study we observed the clinical and microbiological characteristics of children under 15 years admitted to the Central Hospital of the Armed Forces
(HCFFAA) from January 2011 to November 2013 by reviewing medical records are reviewed.

A total of 35 cases with positive urine culture were isolated, where prevalence remains almost similar, with average of 12 cases per year (3\% of hospital admission), it was found the most affected age was 
that of infants under two years old, females predominate in all age groups with a ratio of 4: 1 with regard to males. The most common symptoms were fever and vomiting.

As in other studies the Escherichia coli germ is greater isolation and treatment of choice are the third generation cephalosporin. Three patients have had complications as a result did urosepsis.

Key words: cephalosporin, chronic kidney damage, urosepsis

\section{Introducción}

En los últimos 30-50 años la historia natural de la infección del tracto urinario (ITU) en población pediátrica ha cambiado como resultado de la introducción de los antibióticos y la mejoría de los procedimientos diagnósticos. Estos cambios han contribuido a crear un estado de incertidumbre sobre qué procedimientos diagnósticos y terapéuticos son los más adecuados y como establecer el seguimiento de estos pacientes. El manejo habitual de estos pacientes incluye procedimientos de estudio por imagen, quimioprofilaxis y seguimiento prolongado, que provocan incomodidad en los pacientes y sus familias, así como una utilización excesiva de recursos del Sistema Nacional de Salud (SNS); y todo ello basado en una limitada evidencia (1).

En nuestro entorno existen múltiples documentos sobre la actuación en la ITU en la población pediátrica que abordaron el tema con distintos enfoques, probablemente acordes con las características de la especialidad que los ha propiciado y, en muchos casos, con base en los puntos de vista y opiniones de los autores. El trabajo del Dr. Ochoa et al. (1) es probablemente el más elaborado, desde el punto de vista metodológico, al estar basado en revisiones sistemáticas.

La infección del tracto urinario (ITU) está definida como la invasión, colonización y multiplicación de microorganismos patógenos en el aparato urinario (2) que por lo general ocurre por vía ascendente, siendo las bacterias procedentes de la flora fecal las que colonizan el periné y penetran en la vejiga a través de la uretra. En los niños no circuncidados, estos patógenos procederían de la flora existente bajo el prepucio (3), la posibilidad aunque infrecuente de infección por vía sistémica (hematógena) o directa (cirugías urológicas, traumas abdominales, y otros) (4) son otros mecanismos a ser tomados en cuenta.

Las infecciones del tracto urinario, constituyen una de las infecciones más frecuentes en la atención primaria en salud y en el medio intrahospitalario, es decir, una infección nosocomial. Se pueden presentar a cualquier edad y son más frecuentes en el sexo femenino (5).

La ITU es la entidad clínica que con mayor frecuencia afecta al riñón y a las vías urinarias, con una Prevalencia que oscila entre 0,3 y $7,8 \%$ en la primera infancia; en la edad escolar se ubica entre el 1 y el $3 \%$ para aumentar en los adolescentes con el inicio de las relaciones sexuales (5).

Normalmente la orina es estéril, pudiendo tener gérmenes exógenos, debido a contaminación por su paso a través de la uretra. La aparición de ITU en el ámbito mundial es variable, debido a la edad, sexo, raza, dieta, hábitos higiénicos y sexuales, factores sociales y patogenicidad del germen. Sin embargo, se considera que el sexo más afectado es el femenino, de $20 \%$ a $30 \%$ de las mujeres, por lo menos una vez a lo largo de la vida presentarán ITU, incrementándose esta probabilidad en $1 \%$ 
en cada década de la vida, pudiendo presentar o no sintomatología, el principal germen causal es la Escherichia coli. (3)

Con estos antecedentes, hemos decidido caracterizar la presentación clínica y los aislamientos microbiológicos de las infecciones urinarias en niños y niñas menores de 15 años internados en el Hospital Central de las Fuerzas Armadas (HCFFAA) entre enero de 2011 a Noviembre de 2013.

\section{Materiales y métodos}

Diseño metodológico: El diseño de estudio es del tipo observacional descriptivo retrospectivo de corte transversal.

Población: Un total de 1.138 pacientes admitidos menores de 15 años en el HCFFAA desde enero de 2011 a noviembre de 2013.

Criterios de inclusión: Niños y Niñas menores de 15 años con diagnóstico de Infección del Tracto Urinario, internados en el HCFFAA con Clínica y estudios Microbiológicos desde enero de 2011 a noviembre de 2013.

Criterios de exclusión: Pacientes menores de 15 años con diagnóstico de Infección del Tracto Urinario, con menos de 6 horas de internación y sin estudios microbiológicos desde enero de 2011 a noviembre de 2013.

Muestra: Un total de 35 pacientes reunieron los criterios de inclusión para este estudio.

Procedimiento de la investigación: Procedimiento para le recolección de datos: Se solicitó el permiso correspondiente por escrito al Director de la Institución para informar objetivo de la investigación y su importancia, solicitando el acceso a las historias clínicas según criterio de inclusión.

Se accedió a las historias clínicas y se procedió a extraer los datos relevantes según criterio de inclusión.

Método: Investigación documental, fuente secundaria.

Técnica: Revisión sistemática de historias clínicas seleccionadas según criterios de inclusión.

Instrumento: Base de datos creado con parámetros de estudio para el efecto.

\section{Cuestiones estadísticas:}

procesamiento de los datos se llevó a cabo mediante el sistema Excel $\AA$, las variables y sub-variables fueron expresados en valores absolutos y porcentaje, representado en gráficos analizados con Epiinfo $(\circledR$ con aplicaciones de la estadística descriptiva.

\section{Cuestiones éticas}

Beneficencia: La investigación se realizó con fines estrictamente académicos, por lo que además ayudará a aumentar el nivel de conocimiento, aclarar dudas e implementar acciones a nivel Institucional.

Se espera que con esta investigación se beneficie al sector estudiado y a la profesión médica sobre todo pediátrica, además de proporcionar información para nuevos emprendimientos y ser utilizado por los interesados en el Tema.

Principio de justicia: Se basa en la búsqueda del bien común y en igualdad de condiciones para todos los individuos que llegasen a ser partícipes de la investigación o su resultado, este principio vela por la imparcialidad en la distribución en los beneficios y riesgo. 
Confidencialidad: Los datos obtenidos se mantuvieron en la máxima confidencialidad sin acceso a la identidad de los pacientes, con lo que se aseguró el anonimato de los usuarios.
Resultados

En los tres años de estudio retrospectivo, vemos del total: 1.138 pacientes ingresados, la cantidad de pacientes ingresados al servicio de Pediatría, como los casos de ITU, se han mantenido estables, con un promedio de $3,07 \%$ del total de las internaciones registradas por año (Gráfico 1).

\section{Grafico 1. Estadística hospitalaria de los casos de ITU en el periodo de 2011 a noviembre de 2013}

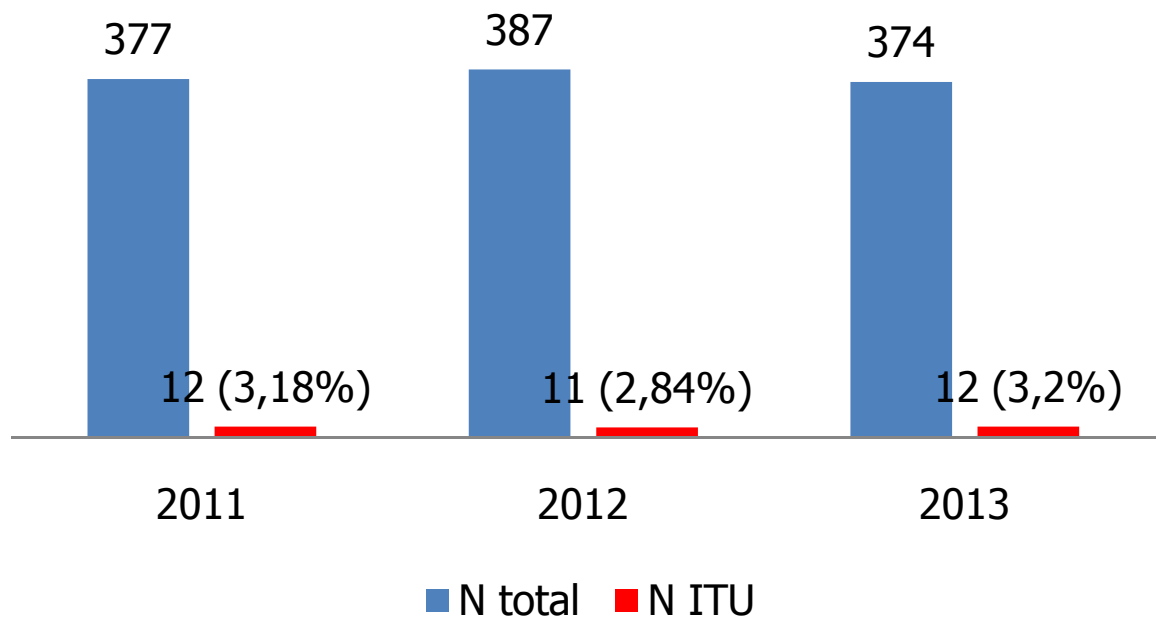

El análisis de los datos segregados por edad y sexo demuestra en general que en todas las edades el sexo femenino es predominante con una relación 4:1.
La edad en la mayoría de los casos se registran entre 1 a 2 años seguido de los niños mayores de 5 años (Gráfico 2).

\section{Grafico 2. Distribución de los pacientes del estudio según edad y sexo}

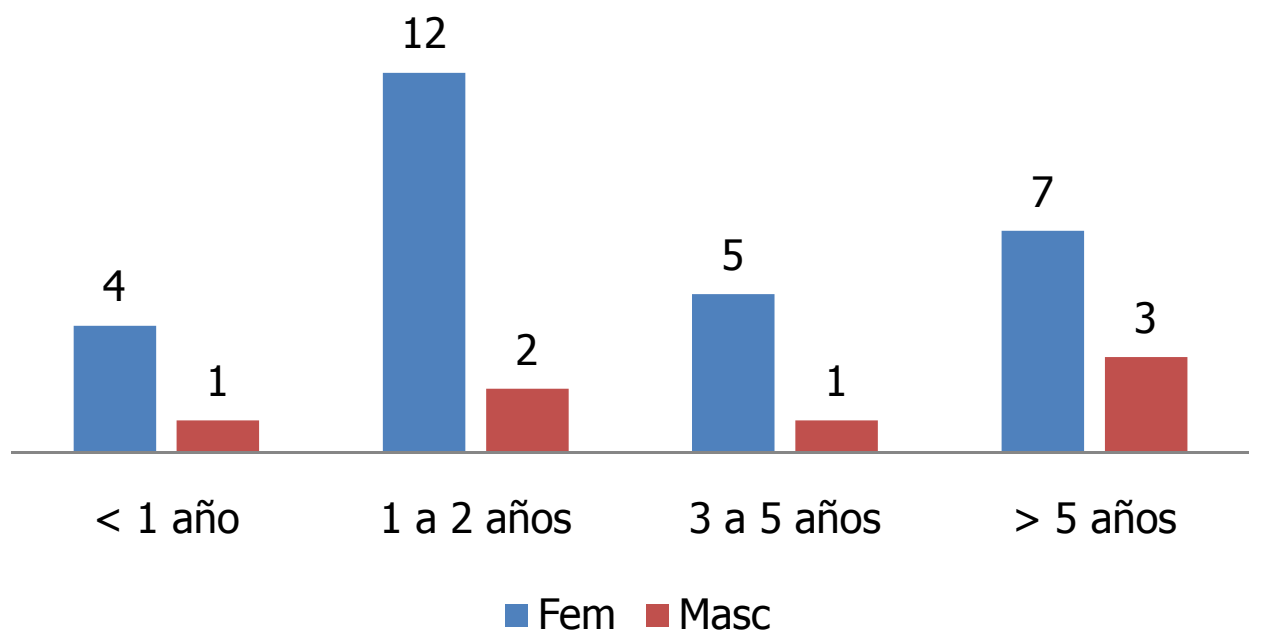


En la serie de estudio, la fiebre se presentó como síntoma clínico de mayor prevalencia con el $71.4 \%$ de los casos en todas las edades. Los vómitos en niños menores de 2 años y dolor abdominal, fue característica presente en escolares y la disuria y hematuria en niños mayores de 5 años (Tabla 1).

Entre los resultados de gabinete, (Tabla 2) resaltan que el $71.4 \%$ presentó ecografía renal normal. La neutrofilia estuvo presente en $54.3 \%$ de los pacientes y la leucocitosis en $22.9 \%$. El hallazgo más llamativo en el análisis simple de orina fue la cantidad de leucocitos por campo que en el $45.7 \%$ superó las 30 células por campo.

Tabla 1. Características clínicas más frecuentes de la infección de las vías urinarias de nuestra población

\begin{tabular}{lrr}
\hline Síntomas & $\mathbf{n}$ & \multicolumn{1}{c}{$\%$} \\
\hline Fiebre & 25 & 71.4 \\
Vómitos & 5 & 14.3 \\
Dolor abdominal & 3 & 8.6 \\
Disuria & 2 & 5.7 \\
Hematuria & 1 & 2.9 \\
\hline
\end{tabular}

Tabla 2. Hallazgos de gabinete en la infección de las vías urinarias de nuestra población

\begin{tabular}{lrr}
\hline Hallazgos de gabinete & $\mathbf{n}$ & $\mathbf{\%}$ \\
\hline Ecografía Renal & & \\
$\quad$ Normal & 25 & 71.4 \\
Alterado & 5 & 14.3 \\
$\quad$ No realizado & 5 & 14.3 \\
\hline Hemograma & & \\
HB $\leq \mathbf{1 1}$ & 13 & 37.1 \\
HTO $\leq \mathbf{3 7}$ & 26 & 74.3 \\
Neutrofilia & 19 & 54.3 \\
Leucocitosis & 8 & 22.9 \\
\hline Urea y Creatinina normal & 35 & 100 \\
\hline Análisis de orina & \multicolumn{3}{c}{} \\
>30 leucocitos por campo & 16 & 45.7 \\
$\quad$ Nitrito (+) & 6 & 17.1 \\
\hline
\end{tabular}

El $88,6 \%$ requirió cambios en el tratamiento de la ITU desde el ingreso al alta, con un 2,9\% de pacientes con profilaxis al alta.

Siete pacientes presentaron ITU a repetición, con $8,6 \%$ de pacientes complicados con Urosepsis (Tabla 3 ).
Tabla 3. Infección de las vías urinarias de nuestra población

\begin{tabular}{lrr}
\hline Factores de riesgo & n & \multicolumn{1}{c}{$\%$} \\
\hline ITU a repetición & 7 & 20.0 \\
Complicaciones: urosepsis & 3 & 8.6 \\
Profilaxis & 1 & 2.9 \\
Cambio de tratamiento & 31 & 88.6 \\
\hline
\end{tabular}

El germen aislado con mayor frecuencia fue Escherichia coli, con el 65,7\% del total de los pacientes, muy por debajo de ese 
porcentaje se encontró a la Klebsiella

pneumoniae con $17,1 \%$ (Grafico 3).

\section{Grafico 3. Microorganismos más frecuentes causantes de las ITU de nuestra serie}

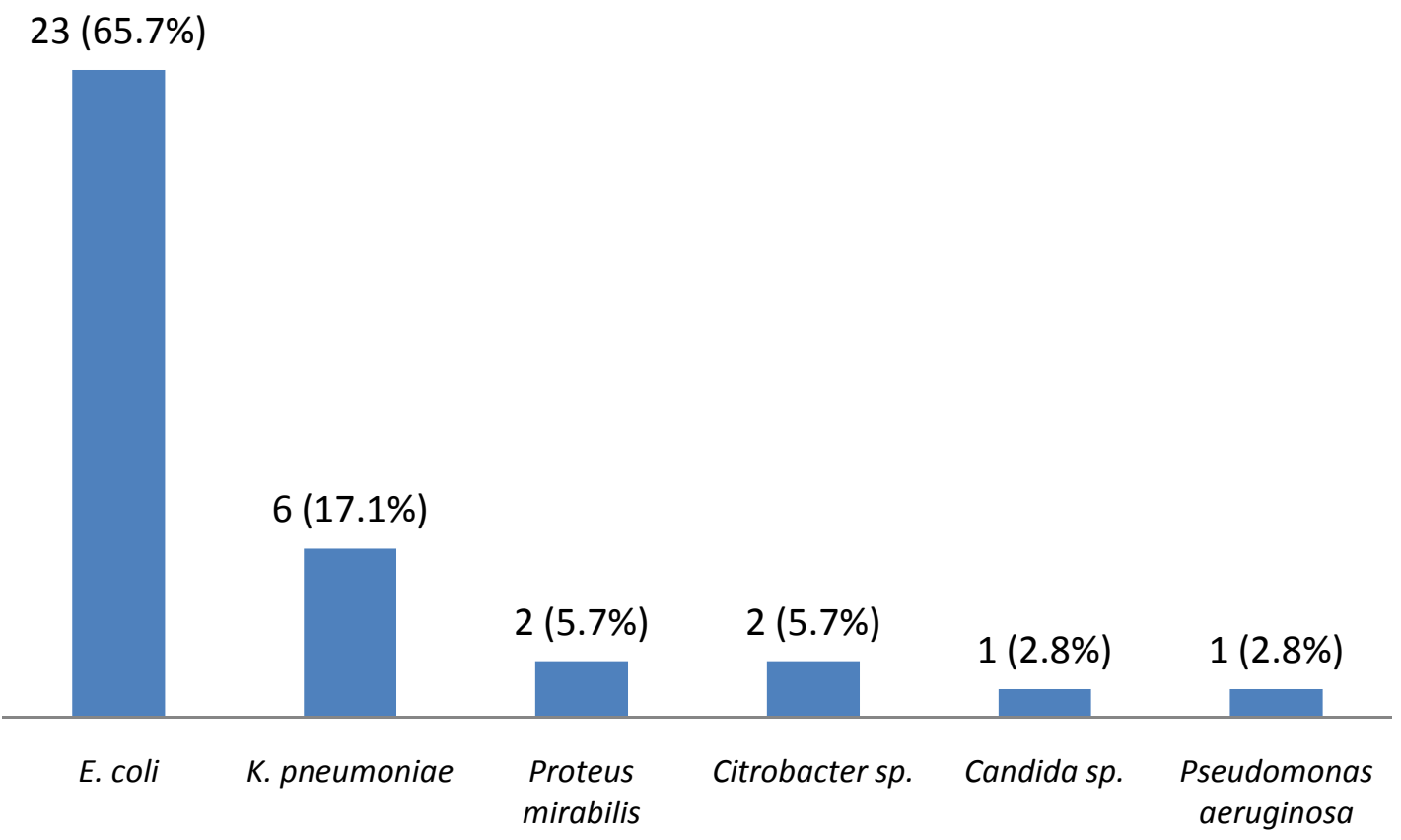

El tratamiento de los pacientes de elección, obteniendo así una frecuencia estudiados, al ingreso, la mayoría ingreso de recambio de tratamiento del $89 \%$ de los con ceftriaxona, con el $52 \%$, cefotaxima 42 pacientes en estudio. $\%$ y al egreso el $89 \%$ de los pacientes fue dado de alta con cefixima como tratamiento

\section{Grafico 4. Tratamiento utilizado al ingreso y al egreso en pacientes con ITU}

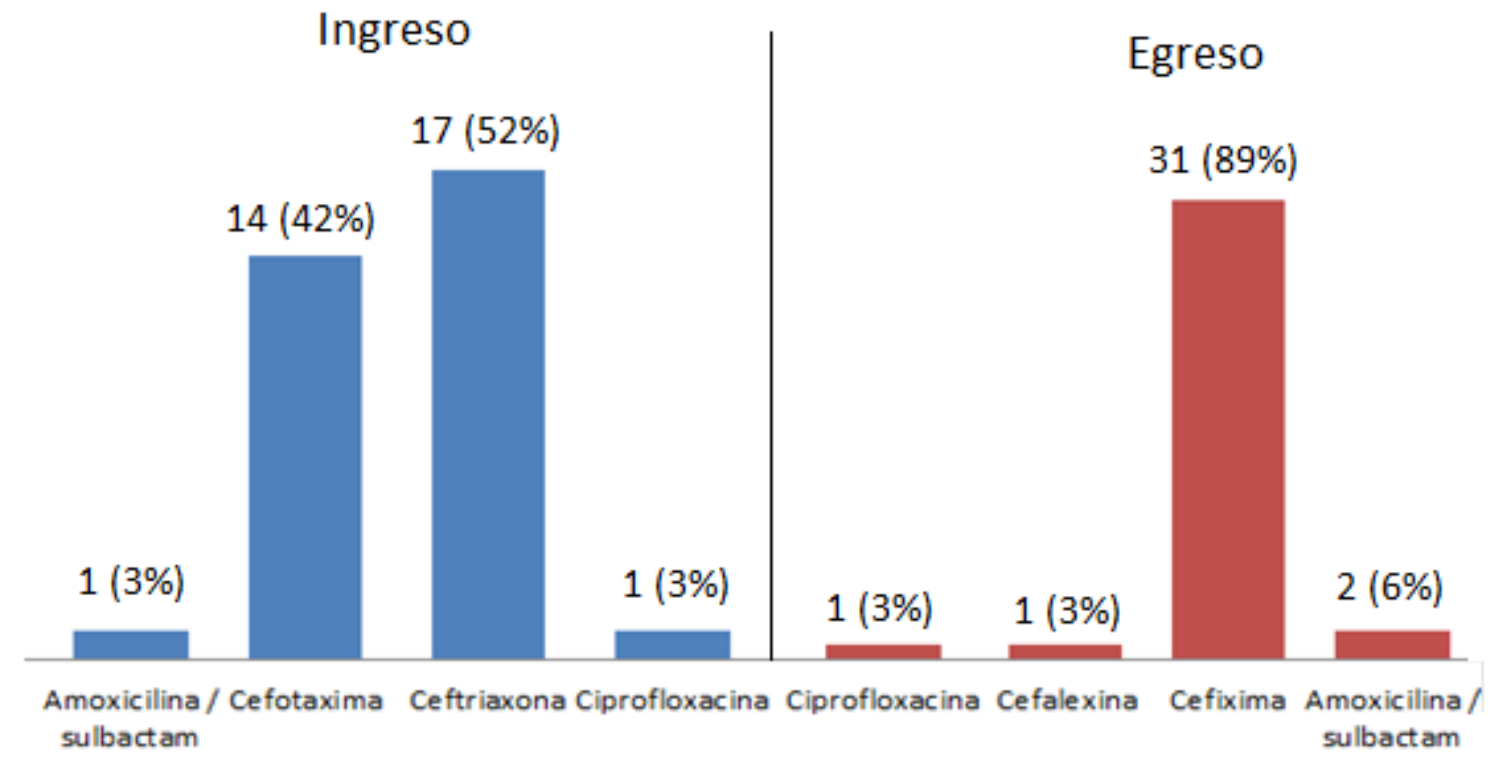




\section{Discusión}

Este estudio demuestra que la frecuencia de la incidencia de infecciones del tracto urinario se mantuvo casi similar a lo largo de los últimos tres años, al igual que la internación por esta entidad en el Servicio de Pediatría del HCFFAA.

Cuando analizamos esos datos y los separamos por edad y sexo vemos que el sexo femenino es predominante con una relación $4: 1$, coincidente en general con otros autores que explican que en el sexo femenino la infección urinaria es más frecuente por la menor longitud de la uretra, la cercanía con gérmenes de la vagina y el ano y el aseo incorrecto de la región perineal (5-7)

En cuanto a la edad se observa que en el período de 1 a 2 años, la infección urinaria se presenta con mayor frecuencia, debido a los factores inmunológicos defensivos aún están inmaduros y coincide con lo observado por otros autores (7).

Los lactantes pequeños generalmente no se presentan con síntomas propios de la vía urinaria, existiendo una pequeña proporción de pacientes que pueden cursar asintomáticos 0 presentar síntomas gastrointestinales, la fiebre fue el factor más frecuente en todas las edades con el $71,4 \%$ de los pacientes de la serie.

Las alteraciones funcionales renales durante el período agudo de infección del tracto urinario pueden ser comprobadas con la realización de la creatinina, la cual nos indica el funcionamiento del filtrado glomerular y generalmente en las infecciones urinarias se mantiene normal (8), tal como fue el resultado hallado en todos los casos estudiados.

Además, debe tenerse en cuenta que no siempre el aumento del número de leucocitos, presente en 80 a $90 \%$ de las infecciones urinarias, es absoluto para el diagnóstico, pudiendo encontrarse en niños febriles con infecciones no localizadas en el tracto urinario o por contaminación vaginal (9), en nuestro estudio, el 45,7 \% de los mismo presentaron un aumento mayor de 30 células por campo en el análisis de orina en fresco.

Entre los factores de riesgo, los episodios de recurrencia pueden ser debidos a recaídas o reinfecciones. Las recaídas son recurrencias por el mismo germen que originó el episodio anterior, dentro de las dos semanas siguientes al término del tratamiento. Las reinfecciones son recurrencias producidas por gérmenes diferentes al que determinó el episodio inicial y se asocian con mayor frecuencia a una enfermedad subyacente del aparato urinario (4), es así como en nuestro estudio hemos encontrado 7 casos de ITU a repetición y tres pacientes con complicaciones, todas ellas urosepsis.

Los bacilos Gram negativos son los principales implicados en las infecciones urinarias pediátricas. Constituyen un grupo complejo formado por varios géneros en los cuales los principales determinantes de virulencia bacteriana están presentes en la mayoría de sus especies.

Escherichia coli es la causante del 80 al $90 \%$ de las infecciones adquiridas en la comunidad y aproximadamente en la mitad de las personas hospitalizadas con factores de riesgo (5) tal como podemos observar en los resultados obtenidos con un porcentaje de retorno positivo para $E$. coli de $65,7 \%$.

En cuanto al tratamiento, tanto al inicio como al egreso, se evaluó que, a pesar de que los aminoglucósidos ocasionan muy baja resistencia en los uropatógenos habituales, pero debido a su toxicidad se limitó su uso. 


\section{Además, debe tenerse en cuenta el Referencias} porcentaje considerable de cepas de $E$. coli que son resistentes a la ampicilina y demás antimicrobianos, lo que trae consigo el empleo cada vez mayor de aminoglucósidos.

\section{Conclusión}

Las niñas menores de dos años son más propensas a adquirir infecciones del tracto urinario.

Junto a la fiebre, el vómito son los síntomas más frecuentes.

La Escherichia coli es el microorganismo con más porcentaje de retorno obtenido en ITU en el grupo estudiado.

La cefalosporina de 3ra generación, tanto por vía oral como endovenosa, es una buena opción para el tratamiento.

La repetición de las ITU y las complicaciones fueron factores de riesgo en el cambio de tratamiento

1. Eiros Bouza JM, Ochoa Sangrador C. Perfil etiológico de las infecciones urinarias y patrón de sensibilidad de los uropatógenos. AnPediatr (Barc). 2007;67(5):461-8

2. Sociedad Argentina de Pediatría (on line) http:://www.sap.org.ar/publicación/

3. Loris Caros y col. Infección urinaria Am J Med .2002,14:165-174.

4. Fernández Javier y col. Infección urinaria. Am J Med 2002,19:127-135.

5. Jhonson C. New Advances in childhood urinary tract infection. Pediatrics in Review 1999;20:335-42

6. Romero Navarro, Benjamin. Infección de vías urinarias en niños. Antibioticos en Pediatría.2008:236-257

7. Grupo de trabajo GPC. Guías de Práctica Clínica en el Sistema Nacional de Salud.Manual metodológico. Madrid:Plan Nacional para el SNS del MSC.Año 2011.

8. Guidelines on Urological Infection. Grabe et alt. European Association of Urology.2011

9. Rubin MI. Examination of the urine in pyelonephritis. Pediatrics 1959; 24,977.July 19.2011

Fecha de recepción: 10 de enero de 2014

Fecha de aceptación: 21 de octubre de 2014

Solicitud de Sobretiros:

Dra. María José Sánchez

Hospital Central de las Fuerzas Armadas

Marijosanchez15@hotmail.com 